\title{
Methods for the detection of gravitational waves from sub-solar mass ultracompact binaries
}

\author{
Ryan Magee, ${ }^{1,2}$ Anne-Sylvie Deutsch, ${ }^{1,2}$ Phoebe McClincy, ${ }^{2}$ Chad Hanna, ${ }^{1,2,3}$ Christian \\ Horst, ${ }^{4}$ Duncan Meacher, ${ }^{1,2,4}$ Cody Messick, ${ }^{1,2}$ Sarah Shandera,,${ }^{1,2}$ and Madeline Wade ${ }^{5}$ \\ ${ }^{1}$ Institute for Gravitation and the Cosmos, The Pennsylvania State University, University Park, PA 16802, USA \\ ${ }^{2}$ Department of Physics, The Pennsylvania State University, University Park, PA 16802, USA \\ ${ }^{3}$ Department of Astronomy and Astrophysics, The Pennsylvania State University, University Park, PA 16802, USA \\ ${ }^{4}$ University of Wisconsin-Milwaukee, Milwaukee, Wisconsin 53201, USA \\ ${ }^{5}$ Kenyon College, Gambier, Ohio 43022, USA
}

(Dated: August 19, 2018)

\begin{abstract}
We describe detection methods for extensions of gravitational wave searches to sub-solar mass compact binaries. Sub-solar mass searches were previously carried out using Initial LIGO, and Advanced LIGO boasts a detection volume approximately 1000 times larger than Initial LIGO at design sensitivity. Low mass compact binary searches present computational difficulties, and we suggest a way to rein in the increased computational cost while retaining a sensitivity much greater than previous searches. Sub-solar mass compact objects are of particular interest because they are not expected to form astrophysically. If detected they could be evidence of primordial black holes $(\mathrm{PBH})$. We consider a particular model of PBH binary formation that would allow LIGO/Virgo to place constraints on this population within the context of dark matter, and we demonstrate how to obtain conservative bounds for the upper limit on the dark matter fraction.
\end{abstract}

\section{INTRODUCTION}

Advanced LIGO [1] and Advanced Virgo's 2] detections of gravitational waves from compact binary coalescences (CBC) have ushered in the dawn of gravitational wave astronomy. To date, there have been 5 detections of binary black hole mergers [3] 6 and 1 detection of a binary neutron star system [7, each of which has expanded our knowledge of the properties and populations of compact objects in our universe. Advanced LIGO and Advanced Virgo's success in detecting traditional sources of gravitational waves suggest that ground based interferometers could be powerful new tools in observing the dark universe. We describe considerations for extensions of traditional compact binary searches to the sub-solar mass regime, and provide motivation for these searches in the context of dark matter. In particular, we consider a model where a uniform distribution of monochromatic primordial black holes $(\mathrm{PBH})$ make up a fraction of the dark matter. We examine the model's robustness and demonstrate how it can place constraints on the abundance of PBHs for different sub-solar mass populations.

\section{ANALYSIS TECHNIQUES}

LIGO compact binary searches rely on matched filtering to extract candidate signals from the noise by correlating known gravitational waveforms with the data. Compact binary searches currently require $\mathcal{O}\left(10^{5}\right)-$ $\mathcal{O}\left(10^{6}\right)$ templates to adequately recover arbitrary signals placed in the parameter spaces considered thus far (binary systems with a total mass of $2 M_{\odot}-600 M_{\odot}[8,9$, . The addition of fully precessing waveforms in future observing runs could increase this by yet another factor of
10, though for now this remains computationally infeasible.

The difficulty of CBC searches scales with both the number and length of the waveforms used as matched filter templates, which could present a problem for subsolar mass searches. Here we focus on the effect of the number of templates in the template bank which is expected to scale (roughly) as:

$$
N \propto m_{\min }^{-8 / 3} f_{\min }^{-8 / 3}
$$

where $m_{\min }$ is the minimum mass included in the search and $f_{\min }$ denotes the starting frequency of the template waveforms [10]. Previous Advanced LIGO searches have searched for binaries with components as light as $1 M_{\odot}$, 8 , 11]; extending these searches to lower masses could easily lead to a $10-100$ time increase in difficulty compared to offline analyses in Advanced LIGO's first observing run. Below we propose increasing $f_{\min }$ to mitigate the increased computational costs associated with low mass extensions of compact binary searches, and we calculate the expected loss in sensitivity that this brings.

\section{A. Estimates of sensitivity}

Second-generation ground-based gravitational wave detectors such as Advanced LIGO and Advanced Virgo are sensitive over a broad range of frequencies $(\sim 10-$ $10000 \mathrm{~Hz}$ ) but they are most sensitive near $100 \mathrm{~Hz}$ [12]. Compact binary pipelines exploit this sensitivity and typically analyze a subset of the total bandwidth. In Advanced LIGO's first observing run, frequencies spanning $10-2048 \mathrm{~Hz}$ were analyzed [13. This is an excellent approximation for standard CBC searches; the majority of the signal-to-noise ratio (SNR) is accumulated at lower 
frequencies and very little sensitivity is lost by cutting the analysis at $2048 \mathrm{~Hz}$. This is an even better approximation for sub-solar mass compact binaries since the frequency evolution of a binary goes as [14:

$$
\dot{f} \propto \mathcal{M}^{5 / 3} f^{11 / 3}
$$

where

$$
\mathcal{M}=\frac{\left(m_{1} m_{2}\right)^{3 / 5}}{\left(m_{1}+m_{2}\right)^{1 / 5}}
$$

is the chirp mass of the system. Sub-solar mass systems therefore are not only long lived, but also spend a long time in LIGO's most sensitive band compared to heavier binaries. This suggests that it may be possible to analyze an even more reduced frequency band than previous searches while retaining a significant amount of SNR.

Since orbital decay is slow for sub-solar mass ultracompact binaries, inspiral only waveforms are a very good approximation of the signal received on earth. The amplitude of the waveform can be written as [15]:

$$
|\tilde{h}(f)|=\frac{1}{D}\left(\frac{5 \pi}{24 c^{3}}\right)^{1 / 2}(G \mathcal{M})^{5 / 6}(\pi f)^{-7 / 6}
$$

and the average recovered signal to noise ratio is given by:

$$
\langle\rho\rangle=\sqrt{4 \int_{f_{\min }}^{f_{\max }} \frac{|\tilde{h}(f)|^{2}}{S_{n}(f)} d f}
$$

where $S_{n}(f)$ denotes the single sided power spectral density, informally referred to as the "noise curve". $f_{\min }$ is determined by either the low frequency noise floor or the starting frequency of the template waveform (whichever is greater) and $f_{\max }$ is determined by the frequency of the innermost stable circular orbit $\left(f_{\mathrm{ISCO}}\right)$ or the ending frequency of the template waveform (whichever is less) where $f_{\mathrm{ISCO}}$ is defined as:

$$
f_{\mathrm{ISCO}}=\frac{c^{3}}{6 \sqrt{6} \pi G M_{\text {total }}}
$$

For a $1 M_{\odot}-1 M_{\odot}$ binary, $f_{\text {ISCO }} \approx 2200 \mathrm{~Hz}$. The frequency monotonically increases for lighter total mass systems; for a sub-solar mass search, $f_{\max }$ is determined by the bandwidth of the template waveforms.

We can substitute the waveform amplitude into the equation for SNR and rearrange to find the horizon distance for a given $\langle\rho\rangle$ (or equivalently, the SNR recovered at some fiducial distance):

$$
D_{\max } \propto \frac{1}{\langle\rho\rangle} \mathcal{M}^{5 / 6} \sqrt{4 \int_{f_{\min }}^{f_{\max }} \frac{f^{-7 / 3}}{S_{n}(f)} d f}
$$

which is dependent on the noise curves, the chirp mass of the binary, and the frequency band of the analysis. This

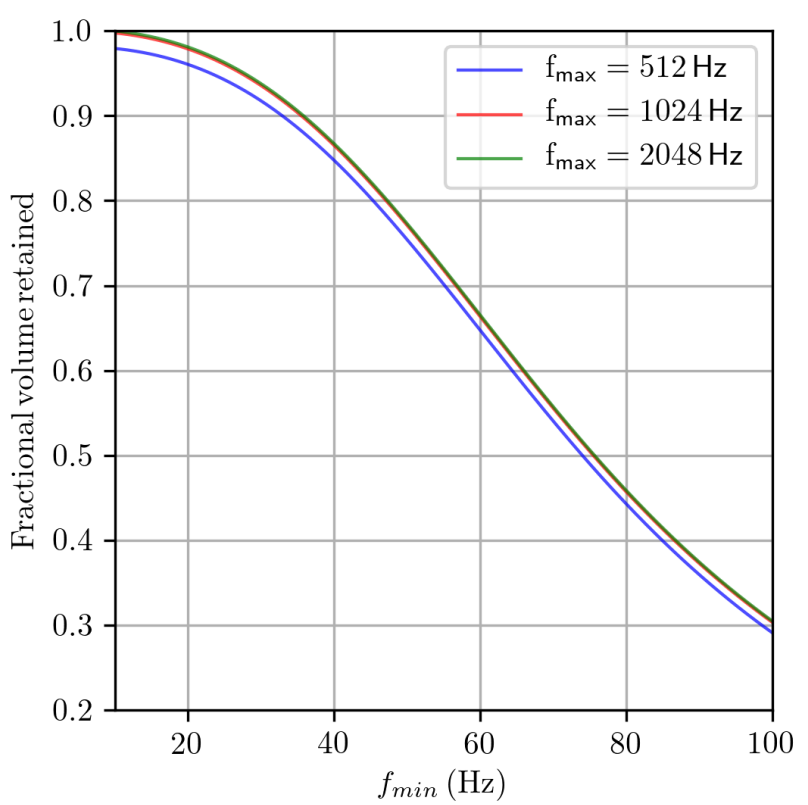

FIG. 1. The fractional volume retained for various values of $f_{\max }$ and as a function of $f_{\min }$. The green, red, and blue lines correspond to upper cut-off frequencies of 2048, 1024, and $512 \mathrm{~Hz}$ respectively. Note that there is very little difference between the various $f_{\max }$ values; this is because there is more than an order of magnitude more noise at these frequencies than the $\sim 100 \mathrm{~Hz}$ region and very little SNR is accumulated there. All values are measured relative to the band $f \in(10 \mathrm{~Hz}, 2048 \mathrm{~Hz})$.

allows us to compare LIGO's sensitivity for frequency bands that do not encompass the full sensitive range. We choose the $f \in(10 \mathrm{~Hz}, 2048 \mathrm{~Hz})$ band as a point of comparison. The fraction of SNR retained is then:

$$
f_{\mathrm{SNR}}=\frac{D\left(f_{\min }, f_{\max }\right)}{D(10 \mathrm{~Hz}, 2048 \mathrm{~Hz})}
$$

Note that this fractional reduction is independent of the mass of the binary. This presents an important trade off in sub-solar mass searches: increasing $f_{\text {min }}$ drives the difficulty of a search down, but it also causes the search to lose sensitivity. This drop in SNR is equivalent to a fractional decrease in LIGO's average range, which means that the observed volume (and therefore the expected number of detections at a given chirp mass) is smaller by a factor of $f_{\mathrm{SNR}}^{3}$. Thus even a $3 \%$ loss in SNR would represent a detection volume nearly $10 \%$ smaller. The sensitive volume retained as a function of $f_{\min }$ and $f_{\max }$ is shown in Fig. 1].

\section{B. Sensitive distance}

Initial LIGO previously carried out searches for compact binaries with components as light as $0.2 M_{\odot}$ [16]. 
Using the relations outlined above and the fact that current Advanced LIGO searches extend to $1 M_{\odot}$ and $f_{\min }=10 \mathrm{~Hz}$, we can estimate the reduction in frequency band and sensitivity required to keep the cost of a subsolar mass search comparable to current Advanced LIGO searches. Equation 1 shows that we expect similar scalings in both $m_{\min }$ and $f_{\min }$. Thus if we decrease the lower mass bound of previous Advanced LIGO searches by a factor of 5 , we need to increase $f_{\min }$ by a factor of 5 as well to keep the number of templates approximately constant. We estimate that in order to modify current searches to extend down to this mass we would need to increase $f_{\min }$ to $\sim 50 \mathrm{~Hz}$. This amounts to a loss of $10 \%$ in SNR and range, and therefore a loss of $\sim 30 \%$ in volume and detection rate. Even with this loss in sensitivity, LIGO would remain incredibly sensitive to sub-solar mass ultracompact binaries.

To emphasize LIGO's improvement in sensitivity even with this reduction in recovered SNR, consider the most recent search for sub-solar mass compact objects carried out in Initial LIGO's third and fourth science runs. The lowest mass binary considered in this search remained visible at a range of $\sim 4 \mathrm{Mpc}$ [17. The estimate outlined here suggests that at the same mass and over a reduced frequency band, Advanced LIGO has a range of $\sim 45 \mathrm{Mpc}$ which corresponds to a sensitive volume more than 1000 times greater. The massive increase in physical volume accessible by Advanced LIGO vastly outweighs any loss in sensitivity due to a moderately reduced frequency band (provided analyzable time remains approximately the same).

\section{Approximation of the merger rate for null-results}

In Equation 7 we defined the horizon distance of the detector. This represents the maximum distance for which an optimally located and oriented source would be recovered with some $\langle\rho\rangle$. In general, however, detectors will measure a weaker response to a gravitational wave depending on the location and orientation of the binary. This reduction is described by the antenna patterns, $F_{+}$ and $F_{\times}$, which always take values less than or equal to 1 and are related to the signal observed on earth through:

$$
h=F_{+} h_{+}+F_{\times} h_{\times}
$$

Averaging the detector response over both location and orientation of the binary reduces the the strain recovered (and therefore the distance to a binary with some fiducial $\langle\rho\rangle)$ by a factor of $2.26[2426$. This can be used to define the average range of the detector as

$$
D_{\text {avg }}=\frac{D_{\max }}{2.26}
$$

The average sensitive distance allows us to approximate limits on the coalescence rate from null results for

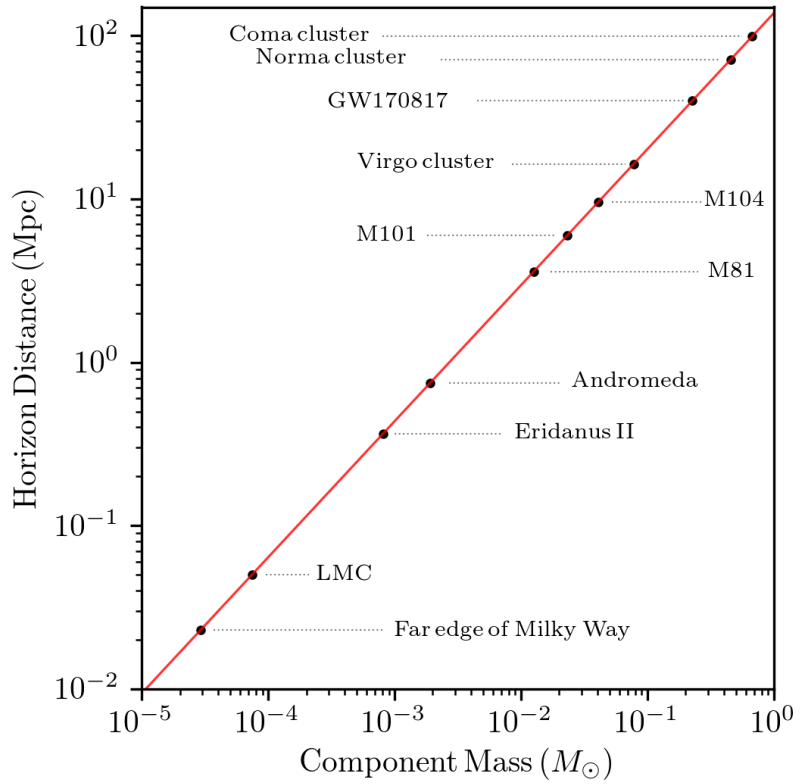

FIG. 2. The distance to an optimally oriented, equal mass binary shown as a function of the component mass. LIGO remains sensitive to $\mathcal{O}\left(10^{-5}-10^{-5} M_{\odot}\right)$ binaries at extragalactic distances. This plot assumes $f_{\min }=10 \mathrm{~Hz}$ and $f_{\max }=2048 \mathrm{~Hz}$ and therefore represents an optimistic view of horizon distance and ignores search difficulty. Astrophysical galaxies, groups, and clusters are included as a reference for cosmological distances. Several objects previously considered as observational candidates for the abundance of dark matter (Eridanus II, LMC/SMC, Segue I) are well within LIGO's range at low masses. Approximate distances taken from [7, 18, 23]. The noise curve used to approximate O1 sensitivity is "Early high/Mid low" from 12.

a general gravitational wave search. The loudest event statistic formalism [27] states that we can constrain the binary merger rate for a specific mass bin, $i$, to $90 \%$ confidence with:

$$
\mathcal{R}_{90, i}=\frac{2.3}{\langle V T\rangle_{i}}
$$

We can estimate the sensitive volume-time for a particular observing run using the earlier range approximation.

$$
\langle V T\rangle_{i}=\frac{4}{3} \pi D_{\text {avg }, i}^{3} T
$$

where $T$ is the analyzable live-time of the two detectors. This method provides an excellent approximation of the sensitive 4 -volume. The remaining plots in this paper use this procedure to estimate LIGO rates and LIGO sensitivity in the sub-solar mass region.

\section{Non-spinning waveforms}

While reducing the frequency band is one way to mitigate the increased computational cost of sub-solar mass 
searches, non-spinning waveforms also offer an easy way to reduce the difficulty by potentially $1-2$ orders of magnitude. There are some theoretical justifications for nonspinning searches: some models predict sub-solar mass black holes to be predominately slowly spinning 28, and LIGO's previous detections have been consistent with low $\chi_{\text {eff }}$ binaries. Regardless, a completely non-spinning binary is clearly a non-physical assumption. The efficacy of using non-spinning waveforms to recover spinning waveforms has been examined before [29 31. In particular, [29] examined neutron star systems and found that nonspinning templates recovered aligned spin binary neutron stars to the desired level only for $-0.2 \lesssim \chi_{\text {eff }} \lesssim 0$.

We performed a similar test on a population of $.5 M_{\odot}-.5 M_{\odot}$ binary black holes. We created a nonspinning template bank covering component masses $m_{i} \in$ $\left(0.3 M_{\odot}, 0.7 M_{\odot}\right)$ using TaylorF2 waveforms [30, 32. We then injected 10000 spinning signals that were purely aligned or anti-aligned with the orbital angular momentum and had dimensionless spin values of $\left|\chi_{i}\right|<0.5$ into fake data. We then calculated the overlap between our non-spinning template waveforms and the spinning signals. We find results similar to those of [29]; at low spin, there is a large overlap between the template waveforms and the injected, spinning signals. At higher spins, however, the maximum overlap rapidly falls off, implying that LIGO would miss a significant fraction of the signals with appreciable spin. In fact, we find that the non-spinning bank used here recovers signals well provided $\chi_{\mathrm{eff}}>-.08$ or $\chi_{\text {eff }}<.02$. As $\chi_{\text {eff }}$ deviates form these values, the fraction of signals missed grows rapidly. A spinning template bank is therefore necessary if sub-solar mass ultracompact binaries are either born with appreciable spin components or accrete enough matter to develop substantial spin. We are currently examining the effects of spin on the computational cost of sub-solar mass CBC searches, as well as other possible ways to mitigate the increased difficulty.

\section{POTENTIAL CONSTRAINTS ON PRIMORDIAL BLACK HOLE ABUNDANCE}

While there is a large population of compact objects below one solar mass, the only objects compact enough for detection by LIGO are black holes and neutron stars. Other compact objects begin to coalesce at too low of an orbital frequency to produce gravitational waves in the sensitive band of ground-based interferometers. Neither black holes nor neutron stars are expected to form below one solar mass via known astrophysical mechanisms, though there are models that propose alternative ways to form black holes at this mass [33, 34]. It is interesting to consider the possibility that sub-solar mass black holes are formed via primordial processes and could be a component of the dark matter. In the event of either a detection or null-result LIGO can provide estimates on the merger rate, so it is therefore necessary to model the bi-

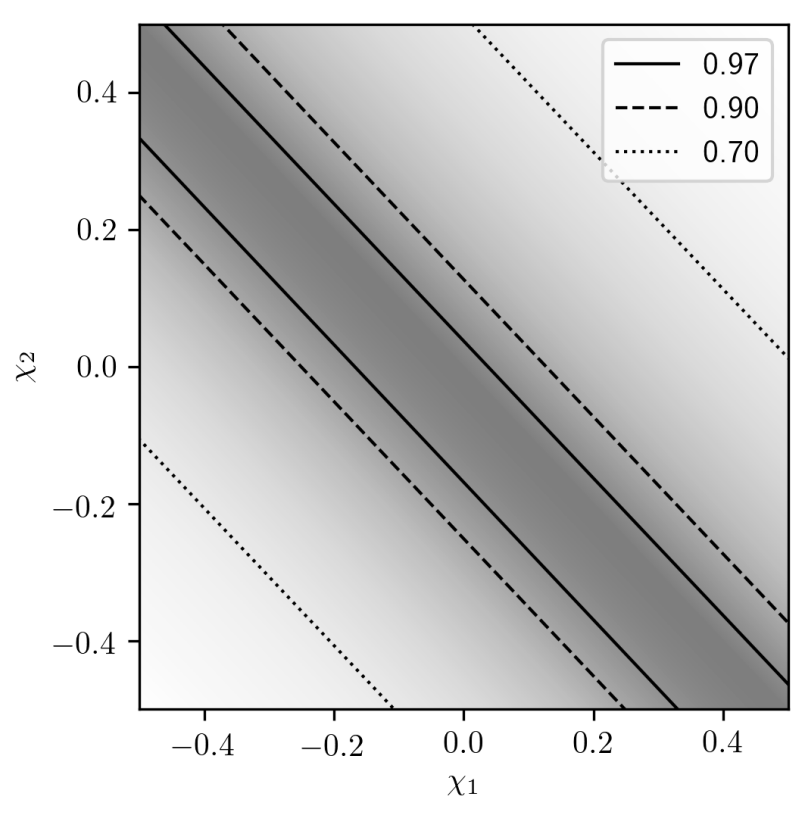

FIG. 3. Recovery of spinning signals with a family of nonspinning template waveforms. Shown in black are lines of constant fitting factor (i.e. the maximum overlap between template waveforms and the injected signals) with the value specified by the line type. The shading shows how the fitting factor changes with the spin of the components in regions between the contours. While systems with $-.084<\chi_{\text {eff }}<.019$ are recovered well, the match between the two waveforms drops rapidly for $\chi_{\text {eff }}$ outside this range. The SNR is proportional to the fitting factor, so the loss in SNR grows rapidly with total spin.

nary formation rate for primordial black holes in order to connect LIGO with primordial populations. Here we describe the sensitivity of one particular model to changes in input parameters, as well as the response of constraints on the dark matter fraction, $f_{\mathrm{PBH}} \equiv \Omega_{\mathrm{PBH}} / \Omega_{\mathrm{DM}}$, to changes in merger rate constraints that could be provided by LIGO. We motivate this model as a way to provide a conservative limit on $f_{\mathrm{PBH}}$.

We consider a model of (initially) uniformly distributed, monochromatic black holes formed in the early universe. A pair of nearest neighbor black holes will start to decouple from the background cosmological expansion and form a binary when the mean energy density in a volume encompassing the two exceeds the background energy density. A third, closest black hole to the binary injects angular momentum in the system by applying tidal forces, which ensures that the two black holes will orbit rather than collide head-on. The resulting expression for the merger rate of primordial black hole binaries in the local universe is given by:

$$
\text { event rate }=\left.n_{\mathrm{PBH}} \frac{d P}{d t}\right|_{t=t_{0}}
$$


where $d P$ is given by:

$$
d P= \begin{cases}\frac{3 f_{\mathrm{PBH}}^{\frac{37}{8}}}{58}\left[f_{\mathrm{PBH}}^{-\frac{29}{8}}\left(\frac{t}{t_{c}}\right)^{\frac{3}{37}}-\left(\frac{t}{t_{c}}\right)^{\frac{3}{8}}\right] \frac{d t}{t}, \quad t<t_{c} \\ \frac{3 f_{\mathrm{PBH}}^{\frac{37}{8}}}{58}\left[f_{\mathrm{PBH}}^{-\frac{29}{8}}\left(\frac{t}{t_{c}}\right)^{-\frac{1}{7}}-\left(\frac{t}{t_{c}}\right)^{\frac{3}{8}}\right] \frac{d t}{t}, \quad t \geq t_{c}\end{cases}
$$

and $n_{\mathrm{PBH}}$ by:

$$
n_{\mathrm{PBH}}=\frac{3 H_{0}^{2}}{8 \pi G} \frac{\Omega_{\mathrm{PBH}}}{M_{\mathrm{PBH}}}
$$

where

$$
t_{c}=Q \alpha^{4} \beta^{7} \bar{x}^{4} f^{25 / 3}
$$

and

$$
\bar{x}=\frac{1}{\left(1+z_{\mathrm{eq}}\right)}\left(n_{\mathrm{PBH}}\right)^{-1 / 3}
$$

with $Q=3 / 170\left(G M_{\mathrm{PBH}}\right)^{-3}, G$ the gravitational constant, $z_{\text {eq }}$ the redshift at matter-radiation equality, and $M_{\mathrm{PBH}}$ the mass of each individual black hole in this population. $\alpha$ and $\beta$ are constants of $\mathcal{O}(1)$ that depend on the dynamics of binary formation and are typically set to 1 . This model has been extensively studied [35-39.

This model provides a direct connection between LIGO and $\mathrm{PBHs}$ via an expected merger rate which is solely a function of the age of the universe, $t_{0}$, given some $M_{\mathrm{BH}}$ and $f_{\mathrm{PBH}}$. The merger rate is not analytically invertible, but if gravitational wave observations provide a constraint on the merger rate for black holes of a particular mass, then it can be numerically solved to obtain an upper limit on $f_{\mathrm{PBH}}$ for that mass bin. Similar procedures have been considered before [37, 39].

It is important to consider the robustness of this model and the relative strictness of the constraints it provides. First, consider the effects of varying $\alpha$ and $\beta$. Numerical simulations suggest that realistic values are $\alpha=0.4, \beta=$ 0.8 [35. Though not immediately evident from the above equation, smaller values of $\alpha$ and $\beta$ lead to larger expected rates and therefore more stringent estimates of the upper limit of $f_{\mathrm{PBH}}$. The dependence of the expected rate on $\alpha$ and $\beta$ is shown explicitly in Figure 4. As $\alpha$ and $\beta$ dip below 1 , the expected merger rate increases. It is a simple extension to approximate how the constraints on $f_{\mathrm{PBH}}$ are affected by variations of $\alpha$ and $\beta$. We can use the procedure outlined in IIC to approximate the upper limit on the merger rate, which we then invert to find limits on $f_{\mathrm{PBH}}$. We present bounds under this approximation for $\alpha=\beta=1$ and $\alpha=0.4, \beta=0.8$ in Figure 5a. This figure shows a general feature of the model: as either $\alpha$ or $\beta$ is decreased, the constraint on $f_{\mathrm{PBH}}$ for a given upper bound on the merger rate becomes tighter. Thus $\alpha=\beta=1$ provides a more conservative limit on $f_{\mathrm{PBH}}$.

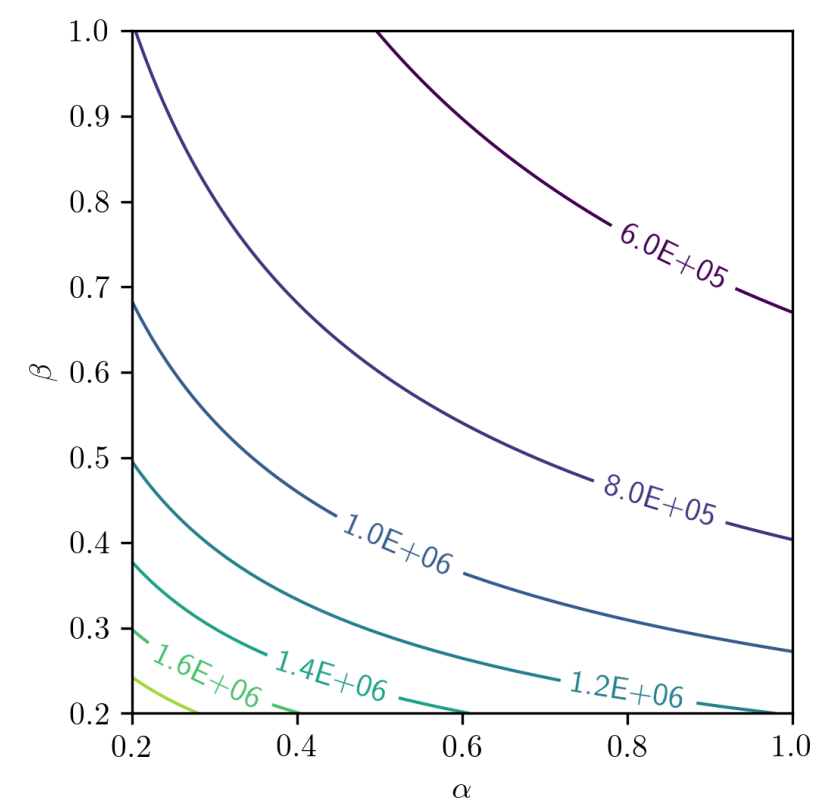

FIG. 4. Merger rate dependence on $\alpha$ and $\beta$ for a fixed dark matter fraction $(f=0.5)$ and primordial black hole mass $\left(M_{\mathrm{BH}}=1.0 M_{\odot}\right)$, shown in units of $\mathrm{Gpc}^{-3} \mathrm{yr}^{-1}$. The expected merger rate strictly increases as either $\alpha$ or $\beta$ are changed from 1.0. Similar behavior is observed independent of the black hole mass or dark matter fraction. This implies that the constraints on the dark matter fraction that are typically published assuming $\alpha=\beta=1$ are conservative for this model.

Of course, allowing $\alpha, \beta$ to increase beyond 1 yields looser constraints. At the time that two PBHs become gravitationally bound to one another, $\alpha$ describes the ratio between the semi-major axis of the binary and the initial physical separation of the two $\mathrm{PBHs}$ at the moment they become bound. It is therefore unphysical to expect $\alpha>1$. $\beta$ helps to determine the minimum ellipticity of the binary; for $\beta>1$, the ellipticity becomes imaginary. $\alpha=\beta=1$ therefore provides the most conservative rate estimate for this model.

Another important consideration is the sensitivity of this model to errors in observational measurements of the merger rate. We can propagate errors in rates measurements through to the dark matter fraction. From our upper limit on the merger rate estimate, we find that $f_{\mathrm{PBH}} \approx .28$ at $0.2 M_{\odot}$ and $f_{\mathrm{PBH}} \approx .04$ at $1.0 M_{\odot}$. If we allow for a $50 \%$ error in the merger rate estimate that this procedure provides we still find $f \in(.17, .37)$ and $f \in(.03, .06)$ for the respective mass bins, thus demonstrating that the constraints are relatively insensitive to even large errors in the upper bound on the merger rate.

There are several other assumptions made in this model that we do not attempt to quantify, but instead provide a brief qualitative argument on their effects. First, we have assumed that primordial black holes are uniformly distributed in space. In reality, we expect 


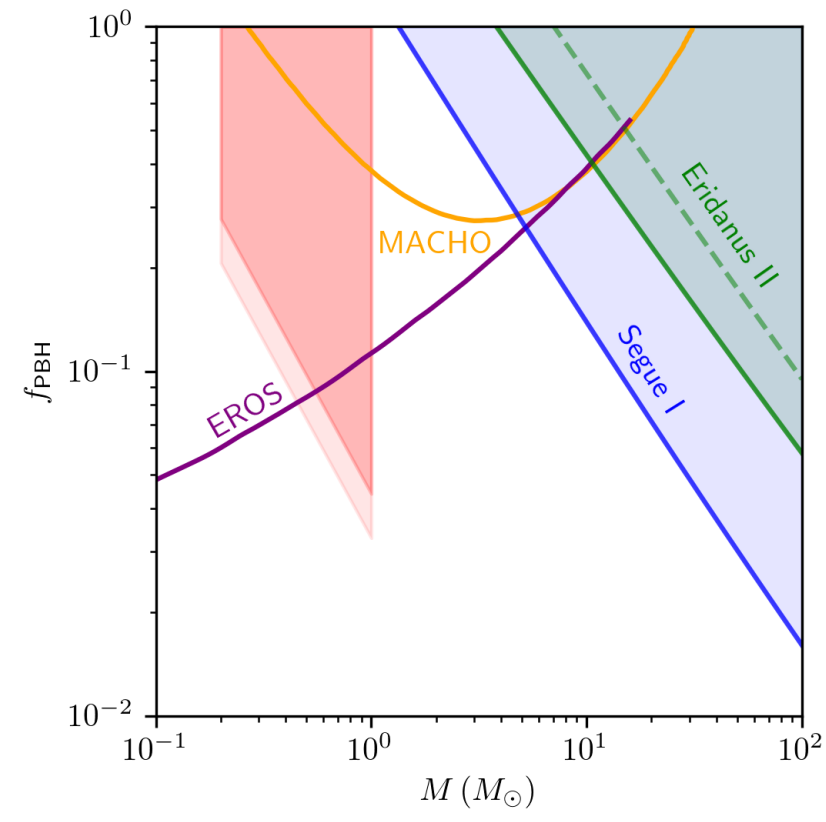

(a) Dark matter constraint dependence on $\alpha$ and $\beta$

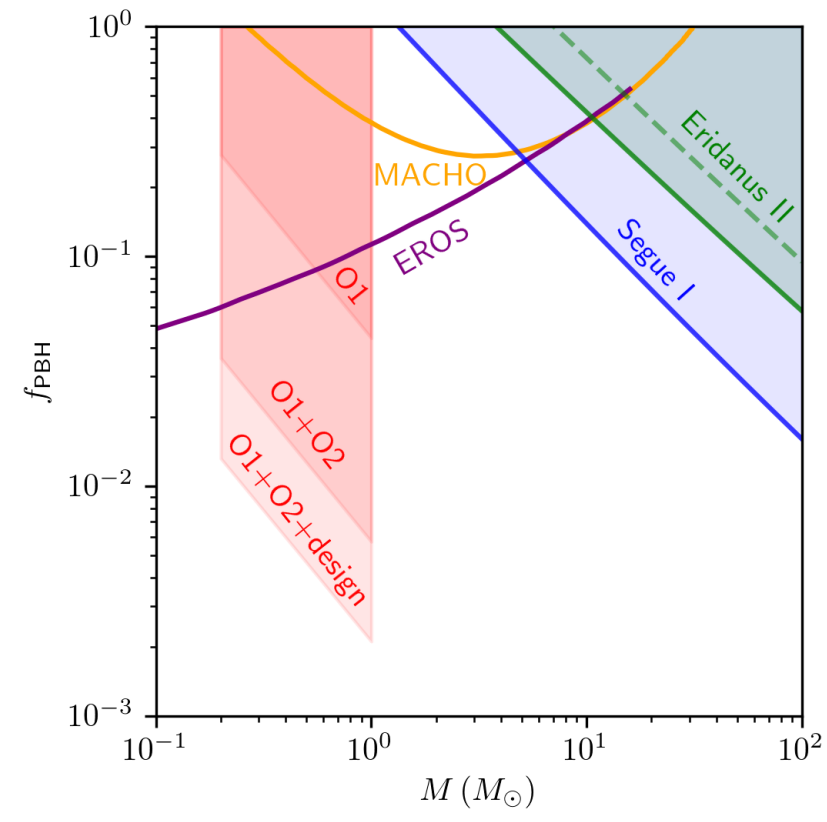

(b) Future outlook for LIGO bounds on PBH dark matter

FIG. 5. 5a Limits on the fraction of dark matter composed of primordial black holes in a monochromatic distribution. Shown in purple, yellow, blue, and green are reproductions of the constraints found in 40,43], respectively. Unlike in [4], the LIGO limits presented here are based on horizon distance estimates using the power spectra and the loudest event statistic [15, 27. This method is described in the text. Potential LIGO results shown in red emphasize the small effect $\alpha$ and $\beta$ have on the constraints. The bottom line shows the limit for $\alpha=0.4, \beta=0.8$, while the upper line shows $\alpha=\beta=1$. $5 \mathrm{~b}$ A possible outlook to the future. Shown here are constraints derived from the same formalism (and assuming continued null results). We follow the procedure mentioned in the text to approximate the rates constraints. Here we assume year long runs operating at $40 \%$ efficiency for the $\mathrm{O} 2$ and design contributions. LIGO will be able to place percent level limits on the fraction of dark matter in PBHs after a year of operating at design sensitivity. The noise curves used for this plot come from the data release associated with [12, specifically the "Early high/Mid low" column for O1, "Mid high/Late low" for O2, and "Design" for design.

PBHs to cluster to some extent which would change the expected event rate for PBH binary mergers. Clustering would tend to increase the amount of binary coalescences, however, so the expected event rate would rise and therefore the maximum permissible fraction, $f_{\mathrm{PBH}}$, would decrease. Therefore a spatially uniform distribution of $\mathrm{PBHs}$ provides a conservative bound on $f_{\mathrm{PBH}}$. We also ignore the binary's evolution between formation and coalescence, as well as the possibility of late-universe binary formation. For a discussion of these effects, which appear to be sub-dominant (though they also drive the expected rate up), see [45. A potentially larger effect comes from the assumption of a purely monochromatic distribution of black holes. Though the framework for this formation model has been extended to the unequal mass case in [35, we have not considered those effects here. Finally, we also ignore the effects of spin on binary formation.

As Advanced LIGO approaches design sensitivity, its horizon distance should increase by a factor of $2-3$ [46]. This, coupled with the more observation, means that LIGO could conceivably have a (cumulative) sensitive $\langle V T\rangle \mathcal{O}(10)$ times larger than was observed in 44. Figure $5 \mathrm{~b}$ shows projections for how continued null results could contribute to constraints on $f_{\mathrm{PBH}}$ for this mass range. Ground based interferometers have the unique ability to strengthen bounds in the sub-solar mass regime by systematics independent of previous microlensing observations [40, 41, 47. This is especially important in light of recent criticisms [48] and studies of the model dependencies of these surveys [49].

\section{FUTURE PROSPECTS AND DISCUSSION}

There are many areas in which sub-solar mass searches can improve on the suggestions outlined here. The most obvious are extensions to lower masses and spinning binaries, each of which presents its own challenges. Lower masses require denser template banks and they persist in LIGO's sensitive band longer. One possible solution could be to alter the width of the frequency band considered for different mass bins, thus stitching together a suitable template bank. Spin is more difficult to incorpo- 
rate; early tests seem to imply at least a factor of 10 more templates would be required for fully spinning binaries. Examining smaller component spins, such as $\chi_{i}<0.3$, could remain computationally feasible and help to mitigate the rapid fall off in sensitivity that non-spinning banks currently experience for moderate to high spin systems. We are actively pursuing extensions in these areas.

More careful $\mathrm{PBH}$ population modeling is also a necessity. In particular, a careful consideration of extended $\mathrm{PBH}$ distributions will offer more accurate and general merger rate predictions. Not only will this allow for more precise constraints, but it will also be useful in examining the feasibility of detecting preferred $\mathrm{PBH}$ distributions peaked in this mass range. While this paper has demonstrated that the model considered typically provides a conservative estimate of the bounds on $f_{\mathrm{PBH}}$, a more general formalism will allow testing of different inflationary models.

\section{ACKNOWLEDGMENTS}

Funding for this project was provided by the Charles E. Kaufman Foundation of The Pittsburgh Foundation. Computing resources and personnel for this project were provided by the Pennsylvania State University. We thank the LIGO CBC working group and John Whelan, Nelson Christensen, and Graham Woan for many useful questions and comments. We thank Kipp Cannon for suggesting we consider non-spinning searches in the mass range considered. This document has been assigned the document number ligo-p1800231-v3.
[1] LIGO Scientific Collaboration, J. Aasi, B. P. Abbott, R. Abbott, T. Abbott, M. R. Abernathy, K. Ackley, C. Adams, T. Adams, P. Addesso, and et al. Advanced LIGO. Classical and Quantum Gravity, 32(7):074001, April 2015.

[2] F. Acernese, M. Agathos, K. Agatsuma, D. Aisa, N. Allemandou, A. Allocca, J. Amarni, P. Astone, G. Balestri, G. Ballardin, and et al. Advanced Virgo: a secondgeneration interferometric gravitational wave detector. Classical and Quantum Gravity, 32(2):024001, January 2015.

[3] B. P. Abbott et al. GW151226: Observation of Gravitational Waves from a 22-Solar-Mass Binary Black Hole Coalescence. Phys. Rev. Lett., 116(24):241103, 2016.

[4] Benjamin P. Abbott et al. GW170104: Observation of a 50-Solar-Mass Binary Black Hole Coalescence at Redshift 0.2. Phys. Rev. Lett., 118(22):221101, 2017.

[5] B.. P.. Abbott et al. GW170608: Observation of a 19solar-mass Binary Black Hole Coalescence. Astrophys. J., 851(2):L35, 2017.

[6] B. P. Abbott et al. GW170814: A Three-Detector Observation of Gravitational Waves from a Binary Black Hole Coalescence. Phys. Rev. Lett., 119(14):141101, 2017.

[7] B. P. Abbott et al. GW170817: Observation of Gravitational Waves from a Binary Neutron Star Inspiral. Phys. Rev. Lett., 119(16):161101, 2017.

[8] B. P. Abbott et al. Binary Black Hole Mergers in the first Advanced LIGO Observing Run. Phys. Rev., X6(4):041015, 2016.

[9] Benjamin P. Abbott et al. Search for intermediate mass black hole binaries in the first observing run of Advanced LIGO. Phys. Rev., D96(2):022001, 2017.

[10] Benjamin J. Owen and B. S. Sathyaprakash. Matched filtering of gravitational waves from inspiraling compact binaries: Computational cost and template placement. Phys. Rev., D60:022002, 1999.

[11] Benjamin P. Abbott et al. Upper Limits on the Rates of Binary Neutron Star and Neutron Star-black Hole Mergers From Advanced Ligo's First Observing run. Astrophys. J., 832(2):L21, 2016.

[12] Benjamin P. Abbott et al. Sensitivity of the Advanced LIGO detectors at the beginning of gravitational wave astronomy. Phys. Rev., D93(11):112004, 2016. [Addendum: Phys. Rev.D97,no.5,059901(2018)].

[13] Cody Messick et al. Analysis Framework for the Prompt Discovery of Compact Binary Mergers in Gravitationalwave Data. Phys. Rev., D95(4):042001, 2017.

[14] Curt Cutler and Eanna E. Flanagan. Gravitational waves from merging compact binaries: How accurately can one extract the binary's parameters from the inspiral wave form? Phys. Rev., D49:2658-2697, 1994.

[15] J. Abadie et al. Sensitivity to Gravitational Waves from Compact Binary Coalescences Achieved during LIGO's Fifth and Virgo's First Science Run. 2010.

[16] B. Abbott et al. Search for gravitational waves from primordial black hole binary coalescences in the galactic halo. Phys. Rev., D72:082002, 2005.

[17] B. Abbott et al. Search for gravitational waves from binary inspirals in S3 and S4 LIGO data. Phys. Rev., D77:062002, 2008.

[18] Simona Mei, John Blakeslee, Patrick Cote, John Tonry, Michael J. West, Laura Ferrarese, Andres Jordan, Eric Peng, Andre Anthony, and David Merritt. The ACS Virgo Cluster Survey. 13. SBF Distance Catalog and the Three-Dimensional Structure of the Virgo Cluster. Astrophys. J., 655:144-162, 2007.

[19] G. Pietrzyński et al. An eclipsing binary distance to the Large Magellanic Cloud accurate to 2 per cent. Nature, 495:76-79, 2013.

[20] F. Vilardell, I. Ribas, C. Jordi, E. L. Fitzpatrick, and E. F. Guinan. The distance to the Andromeda galaxy from eclipsing binaries. Astronomy and Astrophysics, 509:A70, January 2010.

[21] David Carter et al. The HST/ACS Coma Cluster Survey: I - Survey Objectives and Design. Astrophys. J. Suppl., 176:424, 2008.

[22] J. R. Gerke, C. S. Kochanek, J. L. Prieto, K. Z. Stanek, and L. M. Macri. A Study of Cepheids in M81 with the Large Binocular Telescope (Efficiently Calibrated with Hubble Space Telescope). Astrophys. J., 743:176, December 2011.

[23] T. Mutabazi, S. L. Blyth, P. A. Woudt, J. R. Lucey, T. H. Jarrett, M. Bilicki, A. C. Schroder, and S. A. W. Moore. The Norma cluster (ACO 3627) - III. The distance and 
peculiar velocity via the near-infrared Ks-band Fundamental Plane. Mon. Not. Roy. Astron. Soc., 439(4):36663682, 2014.

[24] Lee Samuel Finn and David F. Chernoff. Observing binary inspiral in gravitational radiation: One interferometer. Phys. Rev., D47:2198-2219, 1993.

[25] J. Abadie et al. Predictions for the Rates of Compact Binary Coalescences Observable by Ground-based Gravitational-wave Detectors. Class. Quant. Grav., 27:173001, 2010.

[26] Patrick Sutton. S3 performance of the LIGO interferometers as measured by sensemonitor. 27, 2003.

[27] Rahul Biswas, Patrick R. Brady, Jolien D. E. Creighton, and Stephen Fairhurst. The Loudest event statistic: General formulation, properties and applications. Class. Quant. Grav., 26:175009, 2009. [Erratum: Class. Quant. Grav.30,079502(2013)].

[28] Takeshi Chiba and Shuichiro Yokoyama. Spin Distribution of Primordial Black Holes. PTEP, 2017(8):083E01, 2017.

[29] Hee-Suk Cho and Chang-Hwan Lee. Gravitational Wave Searches for Aligned-Spin Binary Neutron Stars Using Nonspinning Templates. J. Korean Phys. Soc., 72(1):1$5,2018$.

[30] Collin Capano, Ian Harry, Stephen Privitera, and Alessandra Buonanno. Implementing a search for gravitational waves from binary black holes with nonprecessing spin. Phys. Rev., D93(12):124007, 2016.

[31] Tito Dal Canton et al. Implementing a search for aligned-spin neutron star-black hole systems with advanced ground based gravitational wave detectors. Phys. Rev., D90(8):082004, 2014.

[32] P. Ajith, N. Fotopoulos, S. Privitera, A. Neunzert, and A. J. Weinstein. Effectual template bank for the detection of gravitational waves from inspiralling compact binaries with generic spins. Phys. Rev., D89(8):084041, 2014.

[33] Sarah Shandera, Donghui Jeong, and Henry S. Grasshorn Gebhardt. Gravitational Waves from Binary Mergers of Sub-solar Mass Dark Black Holes. 2018.

[34] Chris Kouvaris, Peter Tinyakov, and Michel H. G. Tytgat. Non-Primordial Solar Mass Black Holes. 2018.

[35] Kunihito Ioka, Takeshi Chiba, Takahiro Tanaka, and Takashi Nakamura. Black Hole Binary Formation in the Expanding Universe : Three Body Problem Approximation. Physical Review D, 58(6):063003, 1998.
[36] Takashi Nakamura, Misao Sasaki, Takahiro Tanaka, and Kip S. Thorne. Gravitational waves from coalescing black hole MACHO binaries. Astrophys. J., 487:L139-L142, 1997.

[37] Misao Sasaki, Teruaki Suyama, Takahiro Tanaka, and Shuichiro Yokoyama. Primordial Black Hole Scenario for the Gravitational-Wave Event GW150914. Phys. Rev. Lett., 117(6):061101, 2016.

[38] Yu. N. Eroshenko. Gravitational waves from primordial black holes collisions in binary systems. 2016.

[39] Sai Wang, Yi-Fan Wang, Qing-Guo Huang, and Tjonnie G. F. Li. Constraints on the Primordial Black Hole Abundance from the First Advanced LIGO Observation Run Using the Stochastic Gravitational-Wave Background. Phys. Rev. Lett., 120(19):191102, 2018.

[40] P. Tisserand et al. Limits on the Macho Content of the Galactic Halo from the EROS-2 Survey of the Magellanic Clouds. Astron. Astrophys., 469:387-404, 2007.

[41] R. A. Allsman et al. MACHO project limits on black hole dark matter in the 1-30 solar mass range. Astrophys. J., 550:L169, 2001.

[42] Savvas M. Koushiappas and Abraham Loeb. Dynamics of Dwarf Galaxies Disfavor Stellar-Mass Black Holes as Dark Matter. Phys. Rev. Lett., 119(4):041102, 2017.

[43] Timothy D. Brandt. Constraints on MACHO Dark Matter from Compact Stellar Systems in Ultra-Faint Dwarf Galaxies. Astrophys. J., 824(2):L31, 2016.

[44] B. P. Abbott et al. Search for sub-solar mass ultracompact binaries in Advanced LIGO's first observing run, 2018. arXiv:1808.04771.

[45] Yacine Ali-Haïmoud, Ely D. Kovetz, and Marc Kamionkowski. Merger rate of primordial black-hole binaries. Phys. Rev., D96(12):123523, 2017.

[46] Benjamin P. Abbott et al. Prospects for Observing and Localizing Gravitational-Wave Transients with Advanced LIGO, Advanced Virgo and KAGRA. Living Rev. Rel., 21:3, 2018. [Living Rev. Rel.19,1(2016)].

[47] L. Wyrzykowski et al. The OGLE View of Microlensing towards the Magellanic Clouds. III. Ruling out sub-solar MACHOs with the OGLE-III LMC data. Mon. Not. Roy. Astron. Soc., 413:493, 2011.

[48] M. R. S. Hawkins. A new look at microlensing limits on dark matter in the Galactic halo. Astron. Astrophys., 575:A107, 2015.

[49] Anne M Green. Astrophysical uncertainties on stellar microlensing constraints on multi-Solar mass primordial black hole dark matter. Phys. Rev., D96(4):043020, 2017. 